Article

\title{
Reliability Modelling and Evaluation for LTD System Based on Load-Sharing Model
}

\author{
Jiayun Chu, Tingdi Zhao, Jian Jiao*(D), Zhiwei Chen and Fuchun Ren
}

School of Reliability and Systems Engineering, Beihang University, Beijing 100191, China; bhcjy@buaa.edu.cn (J.C.); ztd@buaa.edu.cn (T.Z.); chenzw@buaa.edu.cn (Z.C.); renfuchun@126.com (F.R.)

* Correspondence: jiaojian@buaa.edu.cn

Received: 25 November 2019; Accepted: 10 December 2019; Published: 16 December 2019

\begin{abstract}
Based on power adding technology, the linear transformer driver (LTD) scheme is widely used to generate high-energy pulsed outputs and adopts a hierarchical and modular structure. Although robust design and fault analysis for basic components have been conducted recently, there is still a lack of enough reliability analysis studies of the whole system. Taking an actual LTD system as an object, this paper presents a system reliability model based on a load-sharing mechanism. A unified load-sharing rule structure is established and four typical rules corresponding to equal, linear, exponential, and local-equal relationships are discussed in detail while evaluating the impact of the load-sharing mechanism. Subsequently, simulation experiments are performed to illustrate the effects of different load-sharing rules as well as analyzing the system reliability in which we simultaneously propose a self-adaptive Monte Carlo simulation flow to achieve the sampling probability adjustment according to the random failure sequence. The simulation results can serve as a suggestion for further improvement of the system reliability. Moreover, the model framework and the simulation analysis method described here are universal and can be applied to evaluate the reliability of other LTD-based systems with tiny modifications.
\end{abstract}

Keywords: LTD system; load-sharing rule; self-adaptive Monte Carlo simulation; reliability analysis

\section{Introduction}

The linear transformer driver (LTD) is a rapidly developing technology used to generate pulsed outputs with the requirement of high voltage, high current, and high power. By contrast with the conventional pulsed-power-accelerator scheme, such as a Marx generator, the LTD system adopts power-adding technology rather than pulse compression and, correspondingly, has several noticeable advantages [1-3]. As a modified induction voltage adder (IVA) [4,5], the LTD also consists of several induction cavities called "LTD stages". A hierarchical and modular structure is adopted in the general design process of the LTD systems [6,7], which decreases the inherent complexity of the system and makes it possible to conduct module maintenance, recycling, and reconfiguration. Aimed to improve the output performance, many research projects focusing on LTD stages have been conducted. Thus, there have been many improved designs, such as Fast LTDs [8,9] and solid-state LTD [10]. All phenomena show that the LTD technology is a hot issue.

With the increasing tendency of the system scale and number of components, the reliability and stability of the LTD system become important problems. Serval fault modes that could affect the pulsed outputs of the LTD system have been discussed in recent research, such as switch self-breakdown and capacitor failure. Robust design and fault analysis for basic components have been conducted recently [11,12], but there is still a lack of studies considering whole system reliability. However, we always need to evaluate whole system reliability to balance the benefits of successful experiments with the serious economic losses and even catastrophic accidents caused by failures. 
Another challenge is that it is dangerous and costly to directly conduct real-world experiments of the LTD system because the experiment is a large-scale cycle of charge and discharge involving numerous capacitors. Therefore, lack of enough experimental data on the whole LTD system, there is an urgent need to make attempts to evaluate LTD system reliability with analytical and/or simulation methods.

As mentioned above, all the LTD-based pulsed-power accelerators adopt a hierarchical and modular structure. Considering the redundancy design, a small number of failure modules are allowed during the experiments. Thus, the LTD system is a typical k-out-of-n: F system [13]. In most reliability analysis for a k-out-of-n: F system, independence is always assumed across the components within the system $[14,15]$. Nevertheless, it is more realistic to consider the feature of fault dependence among the modules during reliability analysis. Load-sharing mechanism can be used to describe the fault dependence, which assumes that the total load will be redistributed into the remaining modules with some rules as there is a module failure. Therefore, the load-sharing k-out-of-n: F system is a more appropriate description of the LTD system.

Since proposed by H.E. Daniels [16] to describe how the strain on yarn fibers increases as individual fibers within a bundle break, the load-sharing mechanism has been wildly considered in the system analysis process at various stages of the life cycle, such as load allocation strategy [17], maintenance analysis [18], and system architecture design [19]. When it comes to reliability analysis, there are also many research works based on load-sharing mechanism. Shao et al. [20] and Liu [21] respectively presented reliability analysis of the load-sharing k-out-of-n system with iid components and non-iid components. A tampered failure rate model and a cumulative exposure model were used to calculate the system lifetime with the assumption that the fixed baseline fault distribution of the components is known in advance [22,23]. Moreover, new failure mechanisms, such as components degradation, were gradually considered [24-26]. Meanwhile, due to the difficulty of acquiring the baseline distribution, Kvam et al. [27] and Park [28] proposed parameter estimation method for the reliability of a load-sharing system.

No matter what areas the load-sharing model has been used in, the most important element of the load-sharing model is the rule that decides how the loads redistribute after some components in the system fail [29]. In this regard, three important load-sharing rules have been considered, consisting of equal, local, and monotone rules [30-32]. However, many researchers only focused on the equal load-sharing rule rather than the remaining two, because it is easy to give a fixed expression of the system reliability while considering the equal load-sharing rule.

In view of the above considerations, the present study defines a unified load-sharing rule structure and analyzes LTD system reliability based on a load-sharing mechanism. Except for the equal load-sharing rule, different failure sequences will lead to different load redistribution results. Considering the randomness of the module failure sequences, a self-adaptive Monte Carlo flow is proposed to simulate the statistic effects.

The rest of the paper is organized as follows. Section 2 describes the LTD system considered and established a system reliability model roughly. In Section 3, we define a unified load-sharing rule structure and discuss four typical rules based on the structure in detail. Subsequently, numerical experiments results are analyzed in Section 4. Finally, Section 5 ends the paper by drawing concluding remarks and summarizing the research paths that deserve most efforts from the community in the near future.

\section{Reliability Model of a Linear Transformer Driver (LTD) System}

\subsection{LTD System Description}

In this paper, we consider a large-scale LTD system with a maximum output current of 50 MA [33]. As shown in Figure 1a, from the perspective of functionality, the LTD system could be divided into two parts, that is the circuit subsystem which stores energy and the trigger subsystem which controls the LTD system. Meanwhile, a five-layer structure is designed to implement the 
pulse-generation mechanism in the circuit subsystem. Specifically, the circuit subsystem can be further divided into 30 branches in parallel. A detailed physical structure of each branch is illustrated in Figure $1 \mathrm{~b}$. We can find intuitively that there are 4 levels parallelly in each branch. As the prime power source, every 30 modules are serially located in one level. Hence the whole system is powered by 3600 modules altogether. Besides, each module comprises 20 identical bricks connected electrically in series, while each brick consists of two capacitors and one switch. Furthermore, the trigger subsystem consists of 720 trigger units, and each trigger unit can generate 10 roads pulse output to control 5 modules. Every 24 trigger units are packaged to fulfill the triggering requirement of different branches. Meanwhile, in order to reduce the impact of trigger unit failure, 5 modules attached to a same trigger unit are distributed in different levels as wildly as possible. Detailed trigger configurations are described in subsequent sections.

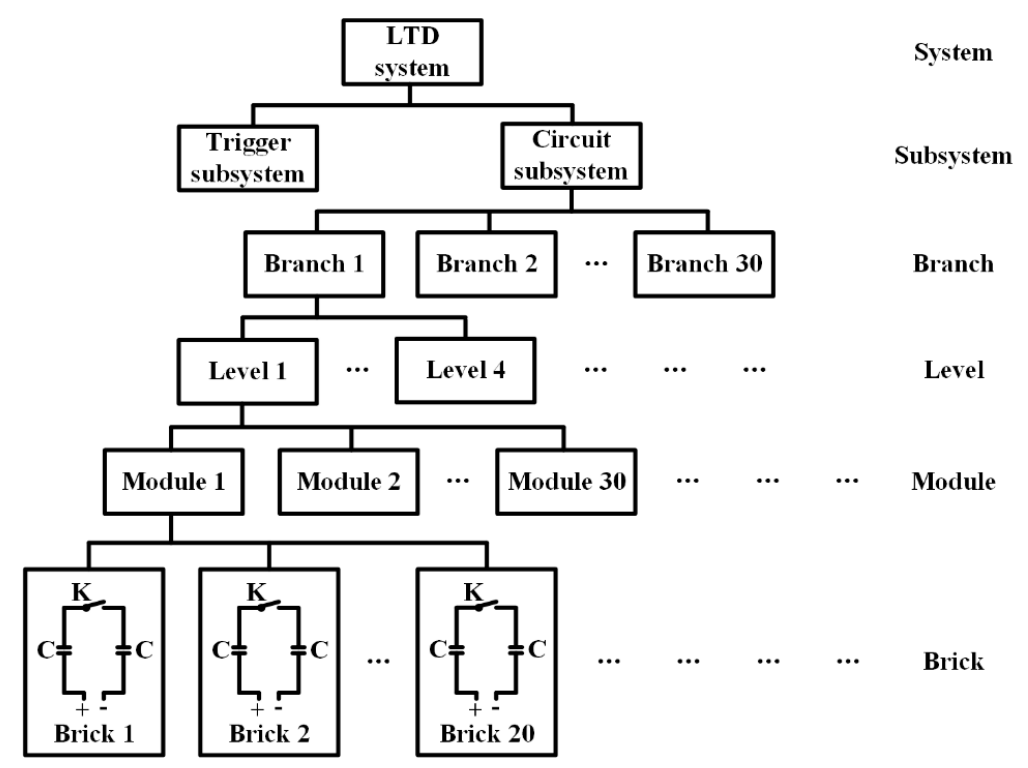

(a)

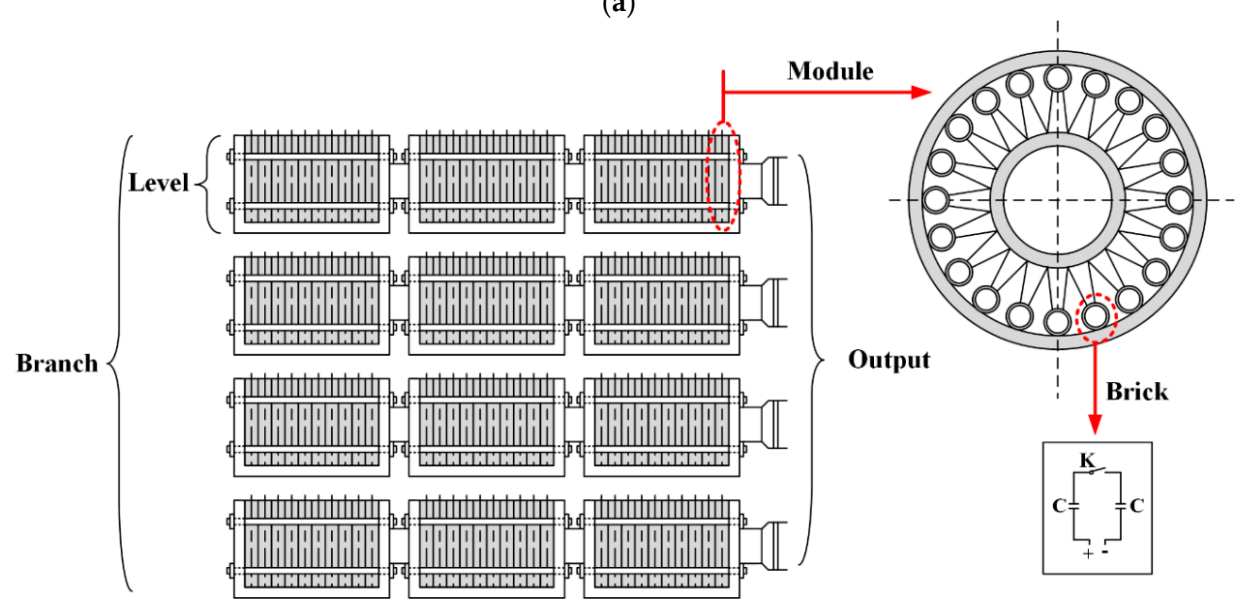

(b)

Figure 1. Description of the linear transformer driver (LTD) system structure. (a) Hierarchical functionality structure diagram; (b) physical structure diagram of the circuit subsystem in the LTD system.

A cycle process of charge and discharge is regarded as the LTD system mission, where the pulsed outputs determine the mission state, namely success or failure. Before switches are triggered, capacitors are charged to a required condition; and when trigger units give the triggering command to the switches in each brick, those switches close and the capacitors synchronously discharge to 
generate the aggregated electric energy. A more detailed introduction of the working mechanism can be found in Liu [34]. Moreover, there is an isolating switch set at the end of each level in the LTD system, which could be switched on only when a pre-specified number of modules in the level are discharged [33]. Because of the existence of the isolating switch, premature voltage outputs caused by a modicum of module failures is superimposed on the remaining normal modules rather that the output load. The isolating mechanism is effective to avoid the circumstance that energy is transferred to the load before the start of the mission caused by a modicum of module failures.

The circuit subsystem is the core energy storage part that determines the output performance. As shown in Figure 1a, the circuit subsystem adopts a five-layer structure, namely subsystem, branch, level, module, and brick. Failures of the basic bricks will have a corresponding impact on the output performance. According to the mission, the fault criteria for different circuit layers are stipulated as follows.

1. Subsystem: any branch failure will cause the subsystem failure.

2. Branch: any level failure in a branch will cause the branch failure.

3. Level: when there are more than $k$ modules failed in a level, the level is judged to fail.

4. Module: any brick failure in a module will cause the module failure.

5. Brick: both the switch self-discharge and the capacitor insulation damage will cause the brick failure, but we only consider the switch self-discharge in the subsequent analysis because it is always the more common reasons.

The trigger subsystem is the auxiliary control part. Regardless of the trigger strategy designed according to different needs, the failure impact mechanism remains unchanged. If both trigger pulses of one module occur earlier than the expected time, the module is paradoxically discharged. Then, the failure will affect the voltages in other modules within the same level, where the voltage redistribution process is subject to the load-sharing mechanism.

\subsection{System Reliability Model}

The pulsed outputs generated by the LTD system are critical to evaluate whether the LTD experiment successes or not. Considering that the circuit subsystem is directly related to the output performance, so we establish the reliability model of the circuit subsystem firstly and then extend it by considering the trigger unit failure. For convenience of description, Table 1 gives some basic notations used in this section.

Table 1. Some basic notations.

\begin{tabular}{ccc}
\hline Order & Name & Abbreviation \\
\hline 1 & circuit subsystem & $\mathrm{c}$ \\
2 & branch & $b_{i}$ \\
3 & level & $l_{i j}$ \\
4 & module & $m_{i j s}$ \\
5 & brick/circuit unit & $u_{i j s t}$ \\
6 & switch & sw \\
\hline
\end{tabular}

Where $i=1,2, \ldots, 30, j=1,2,3,4, s=1,2, \ldots, 30, t=1,2, \ldots, 20$ represent the indexes of the branch, level, module, brick respectively.

According to the hierarchical and modular structure used, the reliability model of the circuit subsystem could be established. Switches self-discharge before the trigger command is a most common cause to influence reliability, which is the core failure mode we considered. Liu [34] and Zeng [35] indicated that the discharge voltage distribution of gas spark switch under thousands of amps current 
circumstance is subject to three-parameter Weibull distribution [36,37], which is applicable to the circumstance in the LTD system, namely:

$$
F(v)=\left\{\begin{array}{cc}
0 & v<\gamma \\
1-\exp \left[-((v-\gamma) / \eta)^{\beta}\right] & v \geq \gamma
\end{array}\right.
$$

where $v$ is the working coefficient here, $F(v)$ is the failure probability, $\eta>0$ is the scale parameter, $\beta>0$ is the shape parameter, and $\gamma>0$ is the location parameter.

If we only focus on the case $v \geq \gamma$, then the switch reliability $R(s w)$ can be calculated by:

$$
R(s w)=1-F(v)=\exp \left[-((v-\gamma) / \eta)^{\beta}\right]
$$

Correspondingly, the initial module reliability $R\left(m_{i j s}\right)$ is:

$$
R\left(m_{i j s}\right)=\prod_{1 \leq t \leq 20} R\left(u_{i j s t}\right)=\exp \left[-20((v-\gamma) / \eta)^{\beta}\right]
$$

The fault criteria show that only are there more than $k$ modules failed in a level, is the level judged to fail. Considering the load-sharing feature, different failure sequences will lead to different load redistribution results. We define the failure sequence $S^{k}$ whose elements are $k$ different integers ranging from 1 to 30 as:

$$
S^{k}=s_{1} s_{2} \ldots s_{k-1} s_{k}, 1 \leq s_{1}, \ldots, s_{k} \leq 30, s_{1} \neq \ldots \neq s_{k}
$$

Then we use $R S^{k}\left(m_{i j s}\right)$ to reflect the modules reliability under the failure sequence $S^{k}$. Specifically speaking, if $s \notin S^{k}, R S^{k}\left(m_{i j s}\right)$ represents the reliability of the module $m_{i j s}$ after all the modules in the sequence $S^{k}$ have failed; if $s \in S^{k}, R S^{k}\left(m_{i j s}\right)$ represents the reliability of the module $m_{i j s}$ after all the modules previous to $s$ in the sequence $S^{k}$ have failed. Now, considering the load-sharing k-out-of-n: F mechanism, the level reliability $R\left(l_{i j}\right)$ can be obtained by:

$$
R\left(l_{i j}\right)=1-\sum_{\forall S^{k}}\left(1-\prod_{s \notin S^{k}} R S^{k}\left(m_{i j s}\right)\right) \prod_{s \in S^{k}}\left(1-R S^{k}\left(m_{i j s}\right)\right)
$$

Subsequently, we can write the branch reliability $R\left(b_{i}\right)$ and the circuit subsystem reliability $R(c)$ as

$$
\begin{aligned}
& R\left(b_{i}\right)=\prod_{1 \leq j \leq 4} R\left(l_{i j}\right) \\
& R(c)=\prod_{1 \leq i \leq 30} R\left(b_{i}\right)
\end{aligned}
$$

When we consider the trigger unit failure, we use the function $T(m)$ to represent a trigger strategy, that is $T\left(m_{i j s}\right)$ represents the corresponding trigger unit connected to the module $m_{i j s}$. Thus, Equation (3) should be modified as:

$$
R\left(m_{i j s}\right)=R\left(T\left(m_{i j s}\right)\right) \prod_{1 \leq t \leq 20} R\left(u_{i j s t}\right)
$$

In summary, the system reliability can be calculated by Equations (1) to (8) except (3). The remaining modules reliability $R S^{k}\left(m_{i j s}\right)$ is the core to solve the problem, which is directly related to the load-sharing rule that we will discuss in the subsequent section. 


\section{Load-Sharing Based Voltage Redistribution Mechanism for Modules in Each Level}

According to the LTD system feature, modules in each level are fault dependent because of the existence of the isolating mechanism. Initially, modules in the same level are loaded with the same voltage according to a pre-specified work coefficient. If any module fails and currently the total number of failure modules is lower than the pre-specified values $k$, the voltage load will be reassigned to the remaining normal modules, which will cause a higher failure probability. The level failure can be regarded as a process of state migration comprising a set of states and a set of transitions [38]. Figure 2 illustrates a failure state migration road. Transitions here are source-action-target triples, which represent the influence of the module failure. There are many failure state migration roads actually corresponding to different failure sequences.

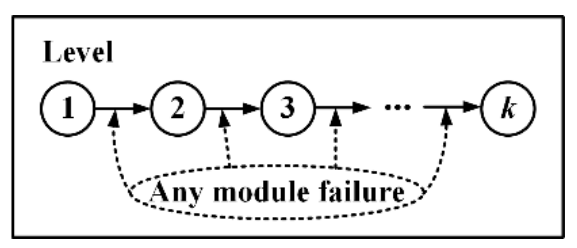

Figure 2. A failure state migration road.

The equal rule is the most commonly considered load-sharing rule. However, the system structure should have effects on the load redistribution process. The closer the normal module is to the failed one, the more likely it will be affected. Thus, it is more realistic to combine the structure factor into the load-sharing rule, such as local and monotone load-sharing rules [31]. Corresponding to the migration process, we assume the voltage of module $p$ at state $x-1$ as $V_{p}(x-1)$. If the failure of module $q$ causes the level to migrate to state $x$ from state $x-1$, the voltage variation of module $p$ at state $x$ can be represented as:

$$
\Delta V_{p}(x)=\left\{\begin{array}{cc}
0 & p \in S_{1}(x) \\
f\left(V_{q}(x-1), D_{p q}\right) & p \in S_{0}(x)
\end{array}\right.
$$

where $S_{0}(x)$ is the set of the normal module at state $x, S_{1}(x)$ is the set of the failure module at state $x$, and $D_{p q}$ is the distance factor related to module $p$ and module $q$ in order to reflect the structure factor. The function $f\left(V_{q}(x-1), D_{p q}\right)$ illustrates that the voltage variation is affected by both the voltage of the failed module and the distance factor. Constraint of the normalization process can be given as:

$$
\sum \Delta V_{p}(x)=\delta \cdot V_{q}(x-1)
$$

where $0<\delta \leq 1$ is an adjustment factor to restrict that the sum of all the voltage variations does not exceed the original voltage of module $q$. Then the voltage of module $p$ at state $x$ can be expressed as:

$$
V_{p}(x)=\left\{\begin{array}{cc}
0 & p \in S_{1}(x) \\
V_{p}(x-1)+f\left(V_{q}(x-1), D_{p q}\right) & p \in S_{0}(x)
\end{array}\right.
$$

Additionally, the function $f\left(V_{q}(x-1), D_{p q}\right)$ can be adjusted according to different rules. Meanwhile, the case $f\left(V_{q}(x-1), D_{p q}\right) \equiv 0$ can cover the traditional reliability analysis process where the load-sharing mechanism is not considered.

Based on the existing discussion of the type of load-sharing rules [30-32,39], in this part, we consider four typical rules corresponding to equal, linear, exponential, and local-equal relationship in detail to reflect common function types, that is linear, non-linear, and segment. The proposed rules cover the generally accepted load-sharing rules. 


\subsection{Equal Load-Sharing Rule}

Equal load-sharing rule is the simplest one, which implies that the load on the failure module will be equally assigned to the remaining normal modules. Thus, the voltage variation $\Delta V_{p}(x)$ can be obtained by:

$$
\Delta V_{p}(x)=\left\{\begin{array}{cc}
0 & p \in S_{1}(x) \\
\frac{\delta \cdot V_{q}(x-1)}{\operatorname{Size}\left(S_{0}(x)\right)} & p \in S_{0}(x) \\
x \geq 1 &
\end{array}\right.
$$

\subsection{Linear Load-Sharing Rule}

The linear load-sharing rule is considered as well. We define that the load on the failure module will be linearly assigned to the remaining normal modules; namely, the nearer the normal element is, the higher voltage variation it will be. Then, we have:

$$
\begin{gathered}
\Delta V_{p}(x)=\left\{\begin{array}{cc}
0 & p \in S_{1}(x) \\
a \cdot\left(D_{\max , q}-D_{p q}+b\right) & p \in S_{0}(x)
\end{array}\right. \\
\text { s.t. } \sum \Delta V_{p}(x)=\delta \cdot V_{q}(x-1) \\
a \geq 0, b \geq 1, x \geq 1
\end{gathered}
$$

where $D_{\max , q}$ denotes the maximal distance between the failure module and normal modules, that is:

$$
D_{\max , q}=\max _{p \in S_{0}(x)}\left\{D_{p q}\right\}
$$

Moreover, $a \geq 0, b \geq 1$ will guarantee the non-negativity of the voltage variation. According to Equation (13), we can obtain:

$$
a=\frac{\delta \cdot V_{q}(x-1)}{\operatorname{Size}\left(S_{0}(x)\right) \cdot\left(D_{\max , q}+b\right)-\sum_{p \in S_{0}(x)} D_{p q}}
$$

Thus, we can write:

$$
\Delta V_{p}(x)=\left\{\begin{array}{cc}
0 & p \in S_{1}(x) \\
\frac{\delta \cdot V_{q}(x-1) \cdot\left(D_{\max , q}-D_{p q}+b\right)}{\operatorname{Size}\left(S_{0}(x)\right) \cdot\left(D_{\max , q}+b\right)-\sum_{p \in S_{0}(x)} D_{p q}} & p \in S_{0}(x)
\end{array}\right.
$$

where $b \geq 1$ represents the adjustment factor.

\subsection{Exponential Load-Sharing Rule}

Similar to the linear load-sharing rule, in the exponential rule, the load on the failure module is distributed to the remaining normal modules according to the distance between them. However, the voltage variation is subject to an exponential relationship. In this condition, we have:

$$
\begin{gathered}
\Delta V_{p}(x)=\left\{\begin{array}{cc}
0 & p \in S_{1}(x) \\
c \times d^{D_{p q}} & p \in S_{0}(x)
\end{array}\right. \\
\text { s.t. } \sum \Delta V_{p}(x)=\delta \cdot V_{q}(x-1) \\
0<d<1, c>0, x \geq 1
\end{gathered}
$$


According to Equation (17), we can obtain:

$$
c=\frac{\delta \cdot V_{q}(x-1) \cdot d^{D_{p q}}}{\sum_{p \in S_{0}(x)} d^{D_{p q}}}
$$

Thus, the voltage variation $\Delta V_{p}(x)$ can be given by:

$$
\Delta V_{p}(x)=\left\{\begin{array}{cc}
0 & p \in S_{1}(x) \\
\frac{\delta \cdot V_{q}(x-1) \cdot d^{D p q}}{\sum_{p \in S_{0}(x)} d^{D p q}} & p \in S_{0}(x)
\end{array}\right.
$$

where $0<d<1$ represents the adjustment factor.

\subsection{Local-Equal Load-Sharing Rule}

Similar to the equal load-sharing rule but more complicated, the local-equal load-sharing rule assumes that the load on the failure module is equally distributed to the remaining normal modules within a limited distance. In this condition, we have:

$$
\begin{gathered}
\Delta V_{p}(x)= \begin{cases}0 & p \in S_{1}(k) \text { or } D_{p q}>f \\
e & p \in S_{0}(x) \text { and } D_{p q} \leq f\end{cases} \\
\text { s.t. } \sum \Delta V_{p}(x)=\delta \cdot V_{q}(x-1) \\
1 \leq f \leq 30, x \geq 1
\end{gathered}
$$

According to Equation (20), we can obtain:

$$
e=\frac{\delta \cdot V_{q}(x-1)}{\operatorname{Size}\left(S_{0}(x) \cap\left\{D_{p q} \mid D_{p q} \leq f\right\}\right)}
$$

Thus, the voltage variation $\Delta V_{p}(x)$ can be obtained by:

$$
\Delta V_{p}(x)=\left\{\begin{array}{cl}
0 & p \in S_{1}(k) \mid D_{p q}>f \\
\frac{\delta \cdot V_{q}(x-1)}{\operatorname{Size}\left(S_{0}(x) \cap\left\{D_{p q} \mid D_{p q} \leq f\right\}\right)} & p \in S_{0}(x) \& D_{p q} \leq f
\end{array}\right.
$$

where $1 \leq f \leq 30$ represents the area influenced by the module failure.

\section{Numerical Simulation and Analysis}

In this section, several simulation experiments are performed to evaluate the impact of the load-sharing mechanism on the module, level and system, respectively. Besides, we also consider the module failure threshold in level and the reliability of the trigger generator unit in the system respectively. Without loss of generality, we refer to the self-breakdown experiment of the switches in Ref. [34] and assume the parameter $\delta=1$. The experimental data are shown in Table 2. 
Table 2. Relationship between working coefficients and failure probability for switch.

\begin{tabular}{cccc}
\hline Working Coefficient (\%) & Failure Probability & Working Coefficient (\%) & Failure Probability \\
\hline 35 & 0 & 70 & $1.40 \times 10^{-5}$ \\
40 & $2.00 \times 10^{-14}$ & 75 & $1.10 \times 10^{-4}$ \\
45 & $3.10 \times 10^{-12}$ & 80 & $7.70 \times 10^{-4}$ \\
50 & $1.70 \times 10^{-10}$ & 85 & $4.40 \times 10^{-3}$ \\
55 & $5.30 \times 10^{-9}$ & 90 & 0.022 \\
60 & $1.00 \times 10^{-7}$ & 95 & 0.098 \\
65 & $1.40 \times 10^{-6}$ & 100 & 0.35 \\
\hline
\end{tabular}

As described in Equation (1), the experimental data above should be subject to a 3-parameter Weibull distribution. We can get the location parameter $\gamma=0.35$, the scale parameter $\eta=0.6815$, the shape parameter $\beta=17.83$. Based on parameters determined, Figure 3 shows the relationship between working coefficient and failure probability of the switch.

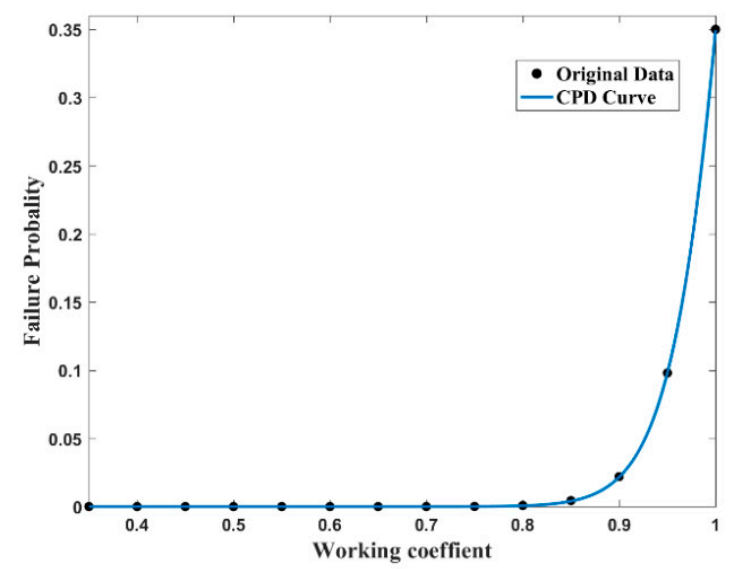

Figure 3. Failure probability of the switch.

Furthermore, considering the randomness of the module failure sequences, Monte Carlo method is adopted to simulate the statistic effects in some parts of analysis.

\subsection{Effect Analysis of Different Load-Sharing Rules on the Modules in a Level}

In Section 2, we have introduced the LTD system in which there are 30 modules in each level. As a typical load-sharing system, the failure probability of the remaining module rises with the increasing voltage load caused by the random failures. Although empirically the linear load-sharing rule may be inferred as the most appropriate rule, we cannot ignore the fact that the current data are too insufficient to determine specific load-sharing parameters. Therefore, we compare four typical rules to show the commonness and differences between them, which is meaningful to help to further identify the detailed rules. Figure 4 presents load redistribution results of different load-sharing rules in different failure cases. The initial working coefficient is set as 0.60 . Moreover, solid lines are used to describe the working coefficients of the normal modules and dotted lines are the continuation of curves. 


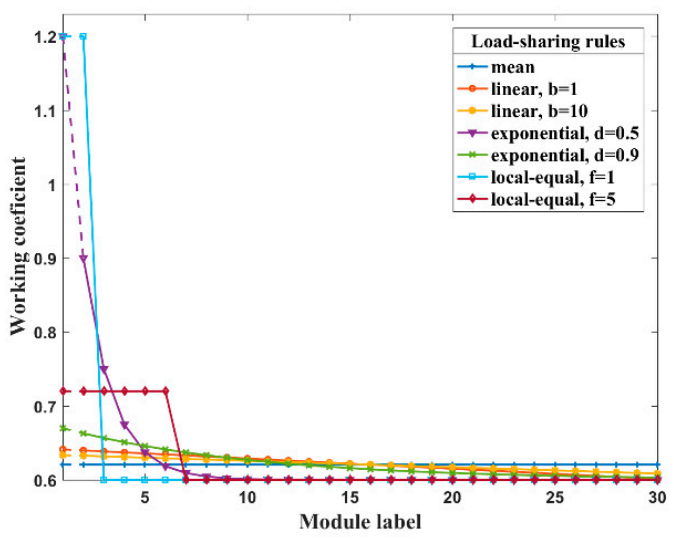

(a)

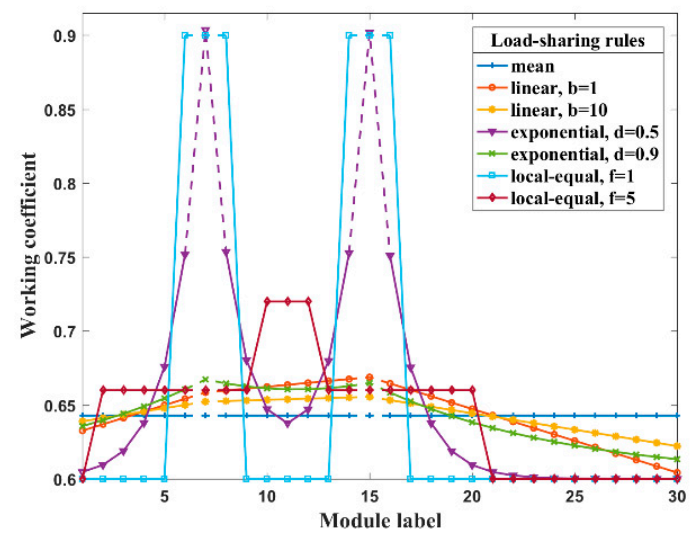

(c)

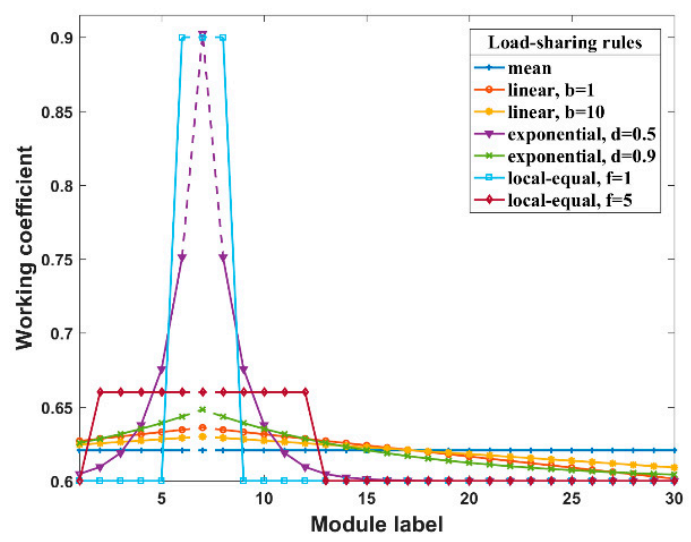

(b)

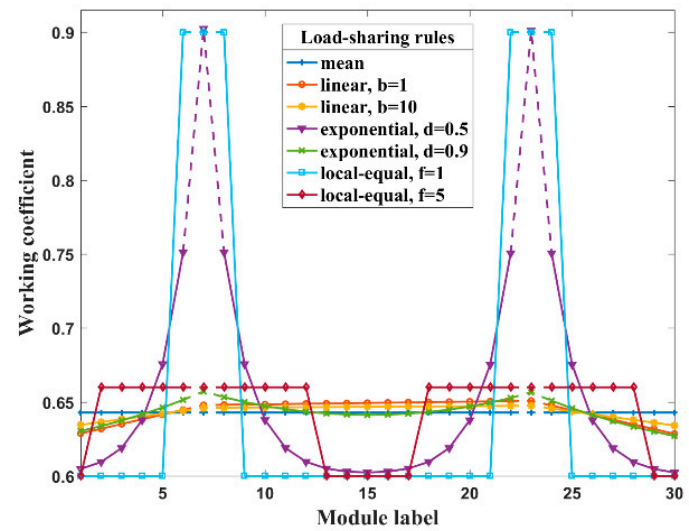

(d)

Figure 4. Load redistribution results of different load-sharing rules under different failure cases. (a) single failure: module \#1; (b) single failure: module \#7; (c) cascading failures: module \#15 after module \#7; (d) cascading failures: module \#23 after module \#7.

Working coefficient curves in the condition that only one module fails are shown in Figure 4a,b. From those graphs, we can clearly see that the coefficient curve of equal rule keeps horizontal while the coefficient curve of local-equal rule is composed of several horizontal segments. As for the linear or exponential rules, the coefficient curve decreases with a special rate centered on the failed module. Furthermore, the impacts of the adjustment factor in different load-sharing rules are also reflected. As shown in Figure $4 a, b$, there is a trend that other rules can be degraded to the equal rule with the adjustment factor increasing. That is, the bigger the parameter $b, d$ or $f$ in the corresponding rule, the smaller the impact of the distance between the normal module and the failed one. Additionally, comparing the coefficient increment sizes in Figure 4a with those in Figure $4 \mathrm{~b}$, it can be seen that the failure of the module around the structure edge tends to cause a larger coefficient change near it.

Working coefficient curves in the condition that there are two failed modules are shown in Figure $4 \mathrm{c}, \mathrm{d}$. The results show that the coefficient curve segment between two failure sources is still subject to a linear relationship in the linear rule, while the same segment seems to be more complicated in the exponential or local-equal rule. The curve of the exponential rule shows a quite obvious fault centrality than the linear rule as before, where the centrality here is used to describe the phenomenon that the failure modules tend to occur within a small local range. The local-equal rule shows differences according to the failed modules. The modules in the overlapping part influenced by different failures will reach a rather high point with the local-equal assumption. 
According to the discussion above, the exponential and local-equal rules show a more obvious fault centrality than the linear rule, while the equal rule assumes all the remaining modules to be the same. Also, the equal rule can be seen as a special case of other rules. Besides, the fault centrality can be broken with the local-equal assumption while the failure occurs in some case.

\subsection{Load-Sharing Based Level Reliability Analysis with the Increasing Number of Failure Modules}

If we only concentrate on the pulsed outputs, a certain number of normal modules in each level can guarantee the output performance to meet the expected requirement [7], which could be easily determined. However, the load-sharing mechanism results in a higher failure rate of each remaining normal modules after some modules have failed unexpectedly. In other words, we will need to bear a greater risk if we continue the LTD experiment when failures occur. Therefore, condition reliability should be evaluated to decide whether to continue the LTD experiment, where the condition reliability here refers that at least one module keeps normal in the experiment when there have been a certain number of failures happened. In this section, we will discuss the module failure threshold $k$ by evaluating the condition reliability.

As discussed before, a different failure sequence leads to a different load redistribution result, which could influence the sampling probability while conducting the Monte Carlo simulation. Therefore, we proposed a self-adaptive Monte Carlo simulation flow to calculate the statistical reliability according to the system structure considered, in which the failures probability for each module used to sample is updated with the failures sequence rather than remaining unchanged. The proposed simulation flow is shown in Table 3.

Table 3. Self-adaptive Monte Carlo simulation flow.

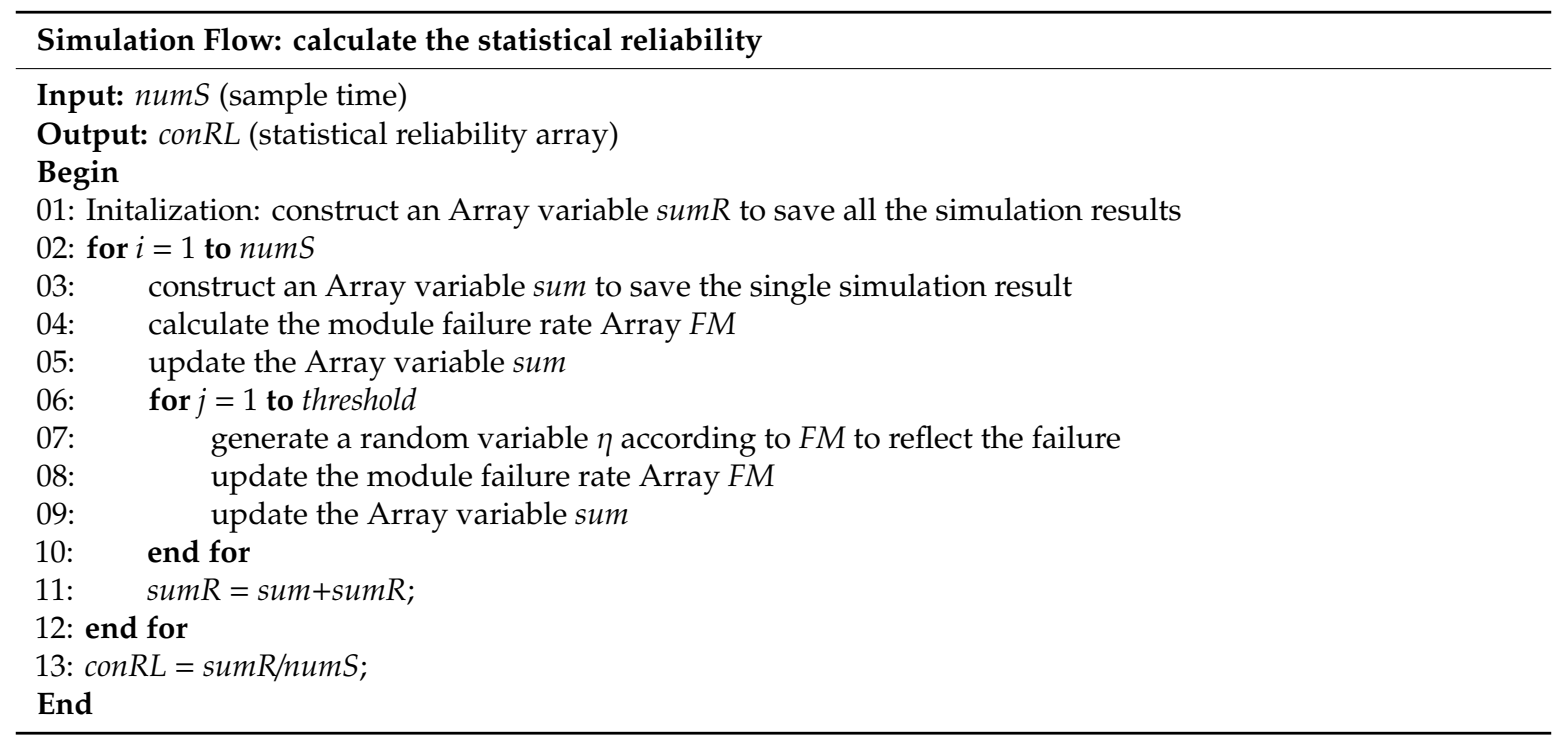

Similarity, we still analyze different types of load-sharing rules. The reliability degradation curve is shown in Figure 5. The results show that there is always a marked fall as long as we consider the load-sharing mechanism, which is caused by the increasing failure rates of the remaining normal modules. Moreover, significant differences can be seen when we compare the different curves. For example, the curve of the equal rule drops sharply after 11 modules failed, while the curve of the exponential rule with the parameter $d=0.9$ declines steadily after 21 modules failed. 


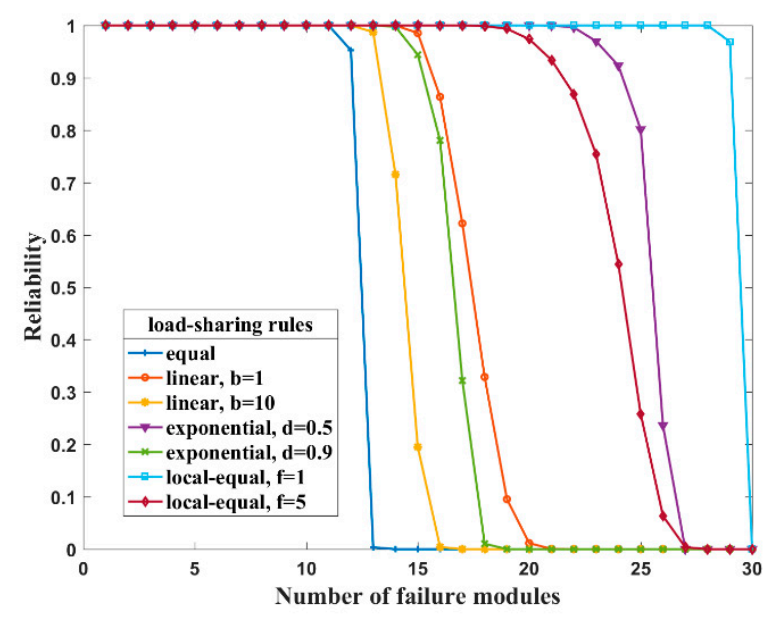

Figure 5. Reliability degradation process of different load-sharing rules.

After further analysis of these differences, we can draw the following conclusions:

1. When just a few modules fail, there are nearly no differences in the impact of different load-sharing rules on the level of condition reliability, namely the early stage of reliability degradation curve is almost overlapped.

2. With the continuously increase of the failure modules, all the reliability degradation curve will drop significantly but the point at which the curve begins to decrease obviously and the corresponding decreasing rate is greatly affected by the load-sharing rule. The more sensitive to the distance factor the load-sharing rule is, the slower the level reliability is degraded. Accordingly, the curve of the equal rule is the first one to decrease with a sharp rate.

3. In the later stage of the reliability curve, almost all the loads are shared by the few remaining modules, thus the failure rates of each module are quite high and the level reliability is close to 0 .

\subsection{System Reliability Analysis with a Special Trigger Strategy Considered}

Load-sharing based reliability analysis has been presented in Section 4.1 and 4.2, both of which focus on the effects of the load-sharing mechanism on the circuit subsystem. In this section, we will further consider the trigger subsystem. Without loss of generality, we choose the linear rule with the parameter $b=1$ as the basic load-sharing mechanism. On the one hand, as shown in Figure 5, the reliability of the linear rule with the parameter $b=1$ falls sharply after 14 modules failed; on the other hand, in terms of the performance of the pulse output, each level can tolerate 5 failure modules at most. Therefore, we set the module failure threshold $k$ as 5 .

Figure 6 presents the current trigger strategy used in the LTD system introduced in Section 2.1. 24 trigger units generate 240 pulse voltages and thus give the trigging command to 120 modules in 1 branch. Specifically, each trigger unit controls the discharge process of 5 modules among which 2 modules belong to a level while the others belong to the remaining level respectively. 


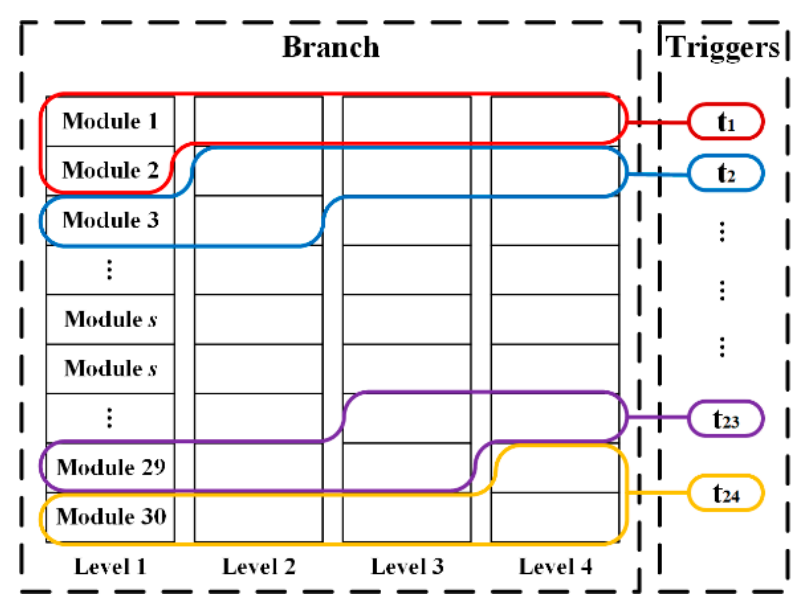

Figure 6. Current trigger strategy.

Figure 7 shows the comprehensive impact of the pre-specified working coefficients and the trigger unit reliability on the LTD system reliability. The horizontal axis represents the pre-specified working coefficients, and the vertical axis represents the LTD system reliability. Besides, different curves are corresponding to different triggering units. As can be seen from Figure 7, when the pre-specified working coefficient is lower than a certain value around 0.65 , the system reliability keeps steady with the increase of the working coefficient and a rather high reliability can be obtained while the trigger unit reliability is more than $1 \times 10^{-3}$; but conversely, the system reliability rapidly declined and it is difficult even almost impossible to keep system reliability at a high acceptable level no matter how high the trigger unit reliability is.

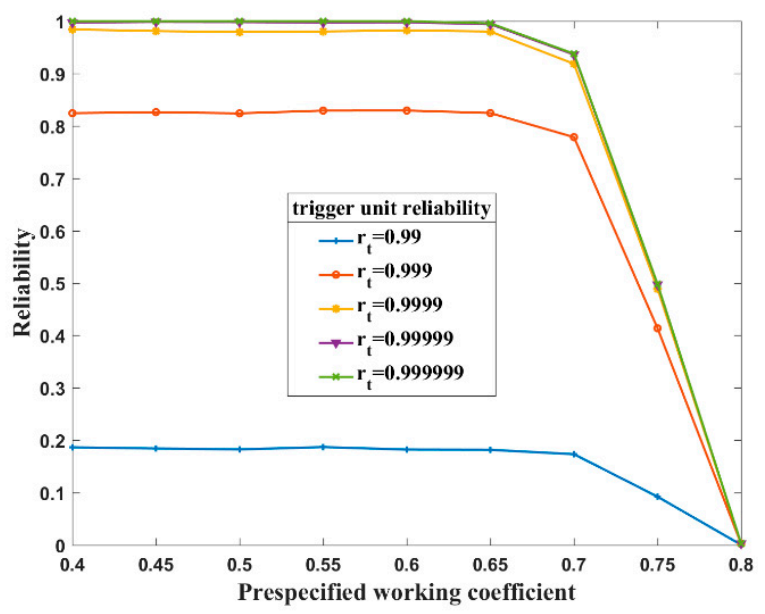

Figure 7. System reliability with the current trigger strategy.

\section{Conclusions}

The purpose of the current study was to conduct LTD system reliability analysis with load-sharing mechanism considered. A unified load-sharing rule structure and a self-adaptive Monte Carlo simulation method are proposed when we evaluate the reliability. With numerical simulation, we have found that the more sensitive to the distance factor the load-sharing is, the more noticeable fault centrality within each level is. Besides, no matter what load-sharing rule we adopt, there is the same trend in the level reliability degradation process, that is, a steady early segment, a rapidly declining middle segment, and a later segment close to zero. But the point at which the level reliability begins to decrease obviously and the corresponding decreasing rate are greatly affected by the load-sharing rule. A load-sharing rule that is sensitive to the distance factor has a slower degradation process. Additionally, we must control the pre-specified working coefficients below a certain value 
as well as selecting a suitable trigger unit in order to obtain considerable system reliability. Those findings contribute in several ways to our understanding of the load-sharing mechanism and have significant implications for the understanding of how the load-sharing mechanism influences LTD system reliability.

In most parts of the discussion, we compare the different impacts among equal, linear, exponential, and local-equal load-sharing rules. Specially, the linear load-sharing rule is used to analyze system reliability with the current trigger strategy considered, which is empirically inferred as an appropriate rule. More experiments will need to be done to find a more appropriate type and the corresponding parameter of the load-sharing rule. Accordingly, the module failure threshold $k$ could be a tradeoff between condition reliability and work efficiency when the load-sharing rule is determined. Notwithstanding these limitations, the present study has an important significance to guide the process of LTD system reliability analysis. We suggest that continued attention is needed to pay for the sake of evaluating and optimizing the LTD system reliability.

Author Contributions: Conceptualization, J.C., T.Z. and J.J.; methodology, J.C., J.J. and F.R.; formal analysis, J.C., and Z.C.; writing — original draft preparation, J.C.; writing — review and editing, J.C. and J.J.; project administration, J.J.; funding acquisition, T.Z. and J.J.

Funding: This research received no external funding.

Conflicts of Interest: The authors declare no conflict of interest.

\section{References}

1. Stygar, W.A.; Cuneo, M.E.; Headley, D.I.; Ives, H.C.; Leeper, R.J; Mazarakis, M.G.; Olson, C.L.; Porter, J.L.; Wagoner, T.C.; Woodworth, J.R. Architecture of petawatt-class z-pinch accelerators. Phys. Rev. Accel. Beams 2006, 10, 536-550.

2. Jiang, W. Review of solid-state linear transformer driver technology. Matter Radiat. Extrem. 2018, 3, 159-164. [CrossRef]

3. Stygar, W.A.; Awe, T.J.; Bailey, J.E.; Bennett, N.L.; Breden, E.W.; Campbell, E.M.; Clark, R.E.; Cooper, R.A.; Cuneo, M.E.; Ennis, J.B.; et al. Conceptual designs of two petawatt-class pulsed-power accelerators for high-energy-density-physics experiments. Phys. Rev. Spec. Top.-Accel. Beams 2015, 18, 110401. [CrossRef]

4. Smith, I.D. Induction voltage adders and the induction accelerator family. Phys. Rev. Spec. Top.-Accel. Beams 2004, 7, 705-707. [CrossRef]

5. Kim, A.A.; Mazarakis, M.G.; Sinebryukhov, V.A.; Kovalchuk, B.M.; Visir, V.A.; Volkov, S.N.; Bayol, F.; Bastrikov, A.N.; Durakov, V.G.; Frolov, S.V.; et al. Development and tests of fast 1-MA linear transformer driver stages. Phys. Rev. Spec. Top.-Accel. Beams 2009, 12, 050402. [CrossRef]

6. Mazarakis, M.G.; Fowler, W.E.; LeChien, K.L.; Long, F.W.; Matzen, M.K.; McDaniel, D.H.; McKee, R.G.; Olson, C.L.; Porter, J.L.; Rogowski, S.T.; et al. High-Current Linear Transformer Driver Development at Sandia National Laboratories. IEEE Trans. Plasma Sci. 2010, 38, 704-713. [CrossRef]

7. Spielman, R.B.; Froula, D.H.; Brent, G.; Campbell, E.M.; Reisman, D.B.; Savage, M.E.; Shoup, M.J.;Stygar, W.A.; Wisher, M.L. Conceptual design of a 15-TW pulsed-power accelerator for high-energy-density-physics experiments. Matter Radiat. Extrem. 2017, 2, 204-223. [CrossRef]

8. Bastrikov, A.N.; Vizir, V.A.; Volkov, S.N.; Durakov, V.G.; Efremov, A.M.; Zorin, V.B.; Kim, A.A.; Kovalchuk, B.M.; Kumpjak, E.V.; Loginov, S.V.; et al. Primary energy storages based on linear transformer stages. Laser Part. Beams 2003, 21, 295-299. [CrossRef]

9. Zhang, P.; Sun, J.; Sun, F.; Qiu, A.; Sun, J.; Hu, Y.; Cong, P. Simulation Analysis of a Pulsed Compact FLTD System for Large-Area Hard X-Ray Sources. IEEE Trans. Plasma Sci. 2016, 44, 803-807. [CrossRef]

10. Collier, L.; Dickens, J.; Mankowski, J.; Neuber, A. Performance Analysis of an All Solid-State Linear Transformer Driver. IEEE Trans. Plasma Sci. 2017, 45, 1755-1761. [CrossRef]

11. Li, J.; Chen, W.; Li, J.; Jiang, W.; Zhong, X.; Gou, Y. Robust Design for Linear Transformer Driver System. IEEE Trans. Plasma Sci. 2015, 43, 3406-3411. [CrossRef]

12. Leckbee, J.J.; Maenchen, J.E.; Johnson, D.L.; Portillo, S.; VanDeValde, D.M.; Rose, D.V.; Oliver, B.V. Design, Simulation, and Fault Analysis of a 6.5-MV LTD for Flash X-Ray Radiography. IEEE Trans. Plasma Sci. 2006, 34, 1888-1899. [CrossRef] 
13. Kapur, K.C.; Lamberson, L.R. Reliability in Engineering Design; John Wiley \& Sons: New York, NY, USA, 1977.

14. Griffith, W.S. Optimal Reliability Modeling: Principles and Applications. Technometrics 2012, 46, 112. [CrossRef]

15. Boddu, P.; Xing, L. Reliability evaluation and optimization of series-parallel systems with k-out-of-n: G subsystems and mixed redundancy types. Proc. Inst. Mech. Eng. Part O J. Risk Reliab. 2013, 227, 187-198. [CrossRef]

16. Daniels, H.E. The statistical theory of the strength of bundles of threads. Proc. A 1945, 183, 405-435.

17. Yamamoto, W.; Jin, L.; Suzuki, K. Optimal allocations for load-sharing k-out-of-n: F systems. J. Stat. Plan. Inference 2009, 139, 1777-1781. [CrossRef]

18. Zhang, N.; Fouladirad, M.; Barros, A. Maintenance analysis of a two-component load-sharing system. Reliab. Eng. Syst. Saf. 2017, 167, 67-74. [CrossRef]

19. Kim, K.O. Optimal number of components in a load-sharing system for maximizing reliability. J. Korean Stat. Soc. 2018, 47, 32-40. [CrossRef]

20. Shao, J.; Lamberson, L.R. Modeling a shared-load k-out-of-n: G system. IEEE Trans. Reliab. 1991, 40, $205-209$. [CrossRef]

21. Liu, H. Reliability of a load-sharing k-out-of-n: G system: Non-iid components with arbitrary distributions. IEEE Trans. Reliab. 1998, 47, 279-284.

22. Jiakai, C.; Yan, H.; Wei, W. Reliability analysis and optimization of equal load-sharing k-out-of-n phased-mission systems. Eksploat. I Niezawodn.-Maint. Reliab. 2015, 17, 250-259. [CrossRef]

23. Amari, S.V.; Bergman, R. Reliability Analysis of k-out-of- $n$ Load-Sharing Systems; Reliability \& Maintainability Symposium: Washington, DC, USA; IEEE: Washington, DC, USA, 2008; pp. 440-445.

24. Yang, C.; Zeng, S.; Guo, J. Reliability Analysis of Load-Sharing K-out-of-N System Considering Component Degradation. Math. Probl. Eng. 2015, 2015, 1-10.

25. Li, Y.; Chen, Y.; Yu, X. A Failure Mechanism Cumulative Model for Reliability Evaluation of a k-out-of-n System with Load Sharing Effect. IEEE Access 2017, 7, 2210-2222.

26. Zhang, J.; Zhao, Y.; Ma, X. A new reliability analysis method for load-sharing k-out-of-n: F system based on load-strength model. Reliab. Eng. Syst. Saf. 2019, 182, 152-165. [CrossRef]

27. Kvam, P.H.; Pe, A.E. Estimating Load-Sharing Properties in a Dynamic Reliability System. J. Am. Stat. Assoc. 2005, 100, 262-272. [CrossRef] [PubMed]

28. Park, C. Parameter estimation for the reliability of load-sharing systems. IIE Trans. 2010, 42, 753-765. [CrossRef]

29. Harlow, D.G.; Phoenix, S.L. The Chain-of-Bundles Probability Model For the Strength of Fibrous Materials I: Analysis and Conjectures. J. Compos. Mater. 1978, 12, 195-214. [CrossRef]

30. Durham, S.D.; Lynch, J.D.; Padgett, W.J.; Horan, T.J.; Owen, W.J.; Surles, J. Localized Load-sharing Rules and Markov-Weibull Fibers. J. Compos. Mater. 1997, 31, 1856-1882. [CrossRef]

31. Suprasad, A.V.; Krishna, M.B.; Hoang, P. Tampered Failure Rate Load-Sharing Systems: Status and Perspectives. In Handbook of Performability Engineering; Springer: London, UK, 2008.

32. Singh, B.; Sharma, K.K.; Kumar, A. Analyzing the dynamic system model with discrete failure time distribution. Stat. Methods Appl. 2009, 18, 521-542. [CrossRef]

33. Ji, C.; Zhou, L.; Jiao, J.; Ren, F.; Chen, L.; Jiang, J.; Zhao, Y. Reliability of large device analysis. High Power Laser Part. Beams 2018, 30, 045003.

34. Liu, X. Breakdown Characteristics of Gas Switch and Its Influence on the Output Parameters of Fast Linear Transformer Driver. Ph.D. Thesis, Xi'an Jiaotong University, Xi'an, China, 2010.

35. Zeng, Z. Introduction to Practical Pulse Technology; Shaanxi Science and Technology Press: Shanxi, China, 2003.

36. Kaminskiy, M.P.; Krivtsov, V.V. A Simple Procedure for Bayesian Estimation of the Weibull Distribution. IEEE Trans. Reliab. 2005, 54, 612-616. [CrossRef]

37. Cousineau, D. Fitting the three-parameter weibull distribution: Review and evaluation of existing and new methods. IEEE Trans. Dielectr. Electr. Insul. 2009, 16, 281-288. [CrossRef] 
38. Pretorius, A.H.; Van Wijk, J.J. Visual analysis of multivariate state transition graphs. IEEE Trans. Vis. Comput. Graph. 2006, 12, 685-692. [CrossRef] [PubMed]

39. Ren, F.; Jiao, J.; Hu, Y.; Zhao, T.; Zhou, L. Reliability Evaluation for Complex Systems with Load Sharing and Failure Dependence: A Case Study on LTD; International Conference on Reliability Systems Engineering: Beijing, China; IEEE, Beijing, China, 2017; pp. 1-6. 